\title{
Maternal noncoding transcripts antagonize the targeting of DNA elimination by scanRNAs in Paramecium tetraurelia
}

\author{
Gersende Lepère, ${ }^{1,2,3}$ Mireille Bétermier, ${ }^{1,2,4}$ Eric Meyer, ${ }^{1,2}$ and Sandra Duharcourt ${ }^{1,2,5}$ \\ ${ }^{1}$ Ecole Normale Supérieure, Laboratoire de Génétique Moléculaire, Centre 75005 Paris, France; ${ }^{2}$ Centre National de la \\ Recherche Scientifique, UMR 8541, 75005 Paris, France
}

\begin{abstract}
The germline genome of ciliates is extensively rearranged during the development of a new somatic macronucleus from the germline micronucleus, after sexual events. In Paramecium tetraurelia, single-copy internal eliminated sequences (IESs) are precisely excised from coding sequences and intergenic regions. For a subset of IESs, introduction of the IES sequence into the maternal macronucleus specifically inhibits excision of the homologous IES in the developing zygotic macronucleus, suggesting that epigenetic regulation of excision involves a global comparison of germline and somatic genomes. ScanRNAs (scnRNAs) produced during micronuclear meiosis by a developmentally regulated RNAi pathway have been proposed to mediate this transnuclear cross-talk. In this study, microinjection experiments provide direct evidence that 25-nucleotide (nt) scnRNAs promote IES excision. We further show that noncoding RNAs are produced from the somatic maternal genome, both during vegetative growth and during sexual events. Maternal inhibition of IES excision is abolished when maternal somatic transcripts containing an IES are targeted for degradation by a distinct RNAi pathway involving 23-nt siRNAs. The results strongly support a scnRNA/macronuclear RNA scanning model in which a natural genomic subtraction, occurring during meiosis between deletion-inducing scnRNAs and antagonistic transcripts from the maternal macronucleus, regulates rearrangements of the zygotic genome.
\end{abstract}

[Keywords: Genome rearrangements; noncoding transcripts; meiotic short RNAs; maternal effects; ciliates; Paramecium]

Supplemental material is available at http://www.genesdev.org.

Received January 23, 2008; revised version accepted March 28, 2008.

The increasing number of non-protein-coding RNAs described has led to speculation that they might be as important as proteins in the regulation of vital cellular functions. In eukaryotes, a variety of specialized RNAi pathways control gene expression at different levels, producing short RNAs that mediate homology-dependent mRNA degradation or translation inhibition, or that regulate chromatin structure through the targeting of epigenetic modifications to specific regions of the genome (Matzke and Birchler 2005; Zaratiegui et al. 2007). In ciliates, a specific class of meiosis-specific short RNAs appears to play a major role in the regulation of the genome-wide rearrangements that occur during de-

Present addresses: ${ }^{3}$ Laboratoire de Biologie Cellulaire, l'Institut National de la Recherche Agronomique (INRA), 78026 Versailles Cedex, France; ${ }^{4}$ Centre de Génétique Moléculaire (CGM), Centre National de la Recherche Scientifique (CNRS) UPR 2167, 1 Avenue de la Terrasse, 91198 Gifsur-Yvette Cedex, France.

${ }^{5}$ Corresponding author.

E-MAIL Sandra.Duharcourt@ens.fr; FAX 33-144323941.

Article is online at http://www.genesdev.org/cgi/doi/10.1101/gad.473008. velopment (Mochizuki and Gorovsky 2004b; Yao and Chao 2005).

These unicellular eukaryotes contain two functionally distinct nuclei. The diploid germline micronuclei are transcriptionally silent during vegetative growth and undergo meiosis during sexual events. The highly polyploid somatic macronucleus $(-800 n$ in Paramecium tetraurelia) is responsible for gene expression throughout the life cycle. The maternal macronucleus is lost after sexual events, while new micro- and macronuclei develop from mitotic copies of the zygotic nucleus. During the development of a new macronucleus, the diploid zygotic genome is endoreplicated and extensively rearranged (Yao et al. 2002). In P. tetraurelia, 50-60 chromosomes are fragmented into 200 shorter macronuclear molecules healed by de novo telomere addition (Aury et al. 2006) as a result of the imprecise elimination of germline-specific regions containing repetitive sequences such as minisatellites and transposons (Le Mouel et al. 2003). Moreover, $\sim 60,000$ short, noncoding internal eliminated sequences 
(IESs) are precisely excised from coding sequences and intergenic regions (Klobutcher and Herrick 1997; Yao et al. 2002). IESs all appear to be single-copy in the haploid genome and are always flanked by two $5^{\prime}$-TA-3' dinucleotides, one of which is retained at the macronuclear junction after excision. They do not share any other strictly conserved motifs, although a degenerate consensus at their extremities (Klobutcher and Herrick 1995) seems to be of importance for the staggered doublestrand breaks that initiate IES excision /Gratias and Betermier 2003). The molecular mechanisms underlying the specific recognition of such a large number of different germline sequences remain poorly understood. In the related ciliate, Tetrahymena thermophila, DNA elimination appears to depend on an RNAi pathway that produces a specific class of short RNAs, 27-30 nucleotides (nt) in length, from the micronuclear genome during meiosis (Mochizuki et al. 2002; Mochizuki and Gorovsky 2004a, 2005; Malone et al. 2005; Lee and Collins 2006). These scanRNAs (scnRNAs) are believed to target histone $\mathrm{H} 3 \mathrm{~K} 9$ and $\mathrm{K} 27$ methylation on germline-specific sequences during macronuclear development, modifications that appear to be necessary for DNA elimination (Taverna et al. 2002; Liu et al. 2004, 2007; Malone et al. 2005; Mochizuki and Gorovsky 2005).

A large body of evidence has shown that genome rearrangement patterns are epigenetically regulated by the maternal macronucleus through homology-dependent effects in Paramecium (Meyer and Duharcourt 1996; Meyer and Garnier 2002; Meyer and Chalker 2007). For instance, transformation of the $P$. tetraurelia vegetative macronucleus with an IES sequence can specifically inhibit the excision of the homologous IES in the new macronucleus of sexual progeny, after meiosis is induced in the transformed clone (Duharcourt et al. 1995). Homology-dependent maternal inhibition of excision was observed for one-third of IESs tested, hereafter called maternally controlled IESs (mcIESs), and varies quantitatively among them (Duharcourt et al. 1998). In the most efficient case, this effect results in the retention of the IES sequence on $100 \%$ of macronuclear chromosome copies, and the altered rearrangement program is maternally inherited in subsequent sexual generations. This gives rise to an epigenetically variant "IES" cell line in which one specific IES is never excised, although its germline genome remains entirely wild type. This and other observations suggest that epigenetic programming of rearrangements involves a transnuclear comparison of the zygotic genome to be rearranged with the previously rearranged genome that is contained in the maternal macronucleus, still present in the cytoplasm at that stage. Very similar homology-dependent effects have been shown to regulate DNA elimination in T. thermophila (Chalker and Yao 1996; Yao et al. 2003; HowardTill and Yao 2006; Kowalczyk et al. 2006), and it has been suggested that scnRNAs mediate the transnuclear comparison of germline and somatic genomes (Mochizuki et al. 2002; Mochizuki and Gorovsky 2004b). ScnRNAs produced by meiotic micronuclei would first be exported to the maternal macronucleus, where they would scan macronuclear DNA by pairing interactions. Those unable to find homologous sequences would then be specifically transported to the developing macronucleus to target the deletion of germline-specific sequences. In $P$. tetraurelia, endogenous short RNAs, $25 \mathrm{nt}$ in length, are also specifically produced from the micronuclear genome during meiosis and may play a similar role since they appear to be required for a subset of rearrangements, including the excision of mcIESs (G. Lepère, M. Nowacki, V. Serrano, J.-F. Gout, S. Duharcourt, and E. Meyer, in prep.). To better account for all known observations, we and others have proposed an alternative scanning model in which micronuclear scnRNAs are compared with an RNA copy of the maternal somatic genome rather than with DNA itself (Garnier et al. 2004; Chalker et al. 2005).

The present study was designed to test the involvement of macronuclear transcripts in the scanning mechanism. We show that noncoding RNAs are indeed produced from the somatic maternal genome, both during vegetative growth and during sexual events. Maternal inhibition of IES excision is abolished when such transcripts containing an IES sequence are targeted for degradation by RNAi, which is mediated in $P$. tetraurelia by 23-nt siRNAs. We further demonstrate by microinjection experiments that a 25-nt synthetic RNA duplex mimicking the structure of scnRNAs actually promotes IES excision. These data provide support for the scnRNA/maternal RNA scanning model, suggesting that epigenetic programming of IES excision is based on a genomic subtraction between deletion-inducing scnRNAs and protective noncoding transcripts from the maternal somatic genome.

\section{Results}

\section{Somatic and germline IES-containing transcripts}

To test the possible role of maternal somatic transcripts in IES excision inhibition, we first investigated transcription of mcIES 51G4404 in an IES $^{+}$cell line, which reproducibly maintains this IES in the macronuclear genome (Duharcourt et al. 1995). The 222-base-pair (bp) IES is located within the coding sequence of the micronuclear G gene (Meyer and Keller 1996), a nonessential surface antigen gene that belongs to a multigene family showing mutually exclusive expression (Caron and Meyer 1989). Large-scale cultures of the IES ${ }^{+}$cell line and of a genetically identical IES ${ }^{-}$cell line, in which the IES is reproducibly excised from the macronuclear genome (Supplemental Fig. S1), were used to prepare total RNA during vegetative growth and at different time points throughout the sexual process of autogamy (selffertilization). Starvation induces meiosis of the micronuclei, and karyogamy gives rise to a diploid zygotic nucleus that divides twice; two of the products differentiate into new micronuclei and the other two into new macronuclei. The maternal macronucleus becomes fragmented shortly after micronuclear meiosis but still contributes $\sim 80 \%$ of total RNA synthesis during the time 
required for development of new macronuclei (Berger 1973). Progression through autogamy was monitored by counting cells at different stages after DAPI staining (Fig. 1). Because cells enter autogamy from a fixed point of the cell cycle (Berger 1986), a minimum asynchrony of 5-6 h is observed between the first and the last cells to undergo meiosis. Transcripts of the $G$ gene could not be detected on Northern blots (data not shown). In contrast, a probe

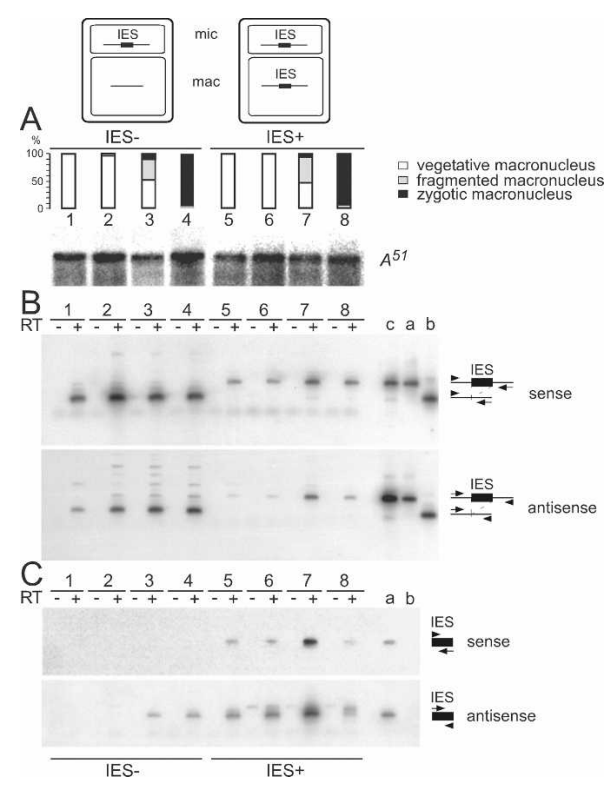

Figure 1. RT-PCR detection of transcripts from both strands of the $G$ gene. The drawings at the top show that the IES ${ }^{+}$cell line only differs from the genetically identical IES ${ }^{-}$cell line by the presence of the 222-bp IES 51G4404 (black box) within its macronuclear genome. $(A)$ Northern blot of RNA extracted at different time points during vegetative growth (lanes 1,5), starvation (lanes 2,6), and autogamy (lanes 3,4,7,8), hybridized with a probe specific for the $A$ surface antigen gene. The histograms indicate, for each time point, the percentage of cells in each cytological stage, as determined by DAPI staining. $(B, C)$ Total RNA was reverse-transcribed with strand-specific primers (arrows) followed by PCR amplification (arrowheads). PCR products were detected by Southern blot analysis using internal PCR fragments as probes. For each sample, the lane marked " -" shows the control reaction without reverse transcriptase. Lanes $a$ and $b$ are control PCR amplifications of IES $^{+}$and IES ${ }^{-}$genomic DNAs, respectively. (B) Detection of sense (top panel) or antisense (bottom panel) G-gene transcripts with RT primers located within the macronuclear sequences flanking the IES. PCR products corresponding to IES-retaining (501 bp) and IES-free (279 bp) transcripts are indicated by symbols on the right. Lane $c$ shows positive controls for the RT reactions using either sense (top panel) or antisense (bottom panel) in vitro transcripts. (Lanes 1-5) The ladder of faint bands visible above the main signal in the bottom panel is due to the presence of tandem repeats in the central part of the $G$-gene coding sequence, so that the RT primer matches at several positions spaced by 222 bp, upstream of the IES. (C) Detection of sense (top panel) or antisense (bottom panel) G-gene transcripts with RT primers located within the IES. Antisense and sense RT-PCR products spanning the entire IES length are indicated by the black rectangles on the right. of the $A$ surface antigen gene revealed abundant mRNAs in all samples (Fig. 1A), indicating that both cultures were expressing that antigen. The presence of low-level $G$-gene transcripts was then tested by a strand-specific RT-PCR assay, using primers located in the coding sequences flanking the IES to easily distinguish the PCR products from IES-containing and IES-free transcripts. Figure $1 \mathrm{~B}$ shows that transcripts initiating outside the IES sequence could be detected in all samples, both on sense (top panel) and antisense (bottom panel) strands. The sizes of the PCR products indicated that transcripts from the IES ${ }^{+}$cell line contained the IES, whereas those from the IES ${ }^{-}$cell line did not, as confirmed by the cloning and sequencing of nine IES ${ }^{+}$and 11 IES $^{-}$cDNAs. We conclude that the maternal macronuclear $G$ gene is transcribed at low levels on both strands, whether or not the IES is excised.

To confirm these results, we used strand-specific RTPCR primers within the IES sequence. We detected IES transcription at all stages of the life cycle in the IES ${ }^{+}$cell line. Both sense and antisense transcripts spanning the entire 222-bp IES were detected (Fig. 1C, lanes 5-8), as confirmed by the sequencing of 16 independent cDNAs (data not shown). Intron-like sequences of 31 and $24 \mathrm{nt}$, typical of Paramecium introns (Aury et al. 2006), were found to be spliced out of one sense and one antisense molecule, respectively. In contrast, no transcription of the IES was detected before autogamy in vegetative or starved cells of the IES ${ }^{-}$cell line (Fig. 1C, lanes 1,2), although antisense transcripts spanning the entire IES sequence appeared specifically during autogamy (Fig. 1C, bottom panel, lanes 3,4). Sense transcripts could also be detected in the same samples when a nested PCR was performed (data not shown). The initial appearance of these developmentally regulated transcripts coincides with the formation of zygotic macronuclei, suggesting they originate from the latter. However, a fraction of them could possibly be produced by meiotic micronuclei and represent scnRNA precursors.

Based on the comparison between the two cell lines, we conclude that, during vegetative growth and starvation, all detectable transcription of the IES and flanking DNA in the IES ${ }^{+}$cell line occurs in the maternal macronucleus, whereas during autogamy, it very likely originates from both maternal and zygotic macronuclei.

\section{Transcription of IESs and maternal regulation}

Only two of the five G-gene IESs (IESs $51 \mathrm{G} 2832$ and 51G4404) are maternally controlled; excision of the other three was shown to be insensitive to the presence of homologous sequences in the maternal macronucleus (Duharcourt et al. 1998). Indeed, when a cloned segment of micronuclear DNA containing the entire $G$ gene (Fig. 2A) was microinjected into the vegetative macronucleus of an IES ${ }^{-}$cell line, at about one copy per haploid genome, Southern blot analysis of post-autogamous progeny showed that IESs 51G4404 and 51G2832 were maintained in $100 \%$ and $65 \%$ of macronuclear chromosome copies (Fig. 2B), while other IESs were completely ex- 


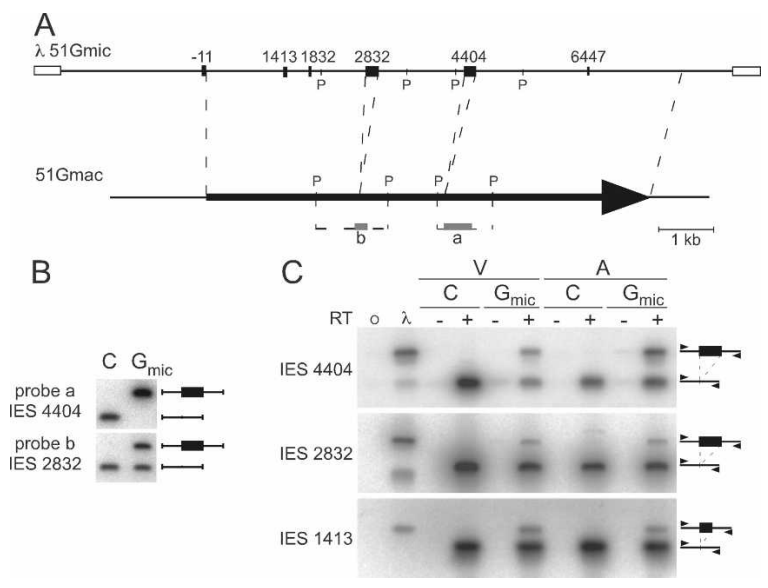

Figure 2. Transcription of different IESs after transformation of the macronucleus with the micronuclear $G$ gene. $(A)$ Maps of the macronuclear $G^{51}$ gene $(51 \mathrm{Gmac})$ and of the insert of phage $\lambda 51 \mathrm{Gmic}$, carrying the micronuclear version of the $G^{51}$ gene. IESs are shown as black boxes in the micronuclear sequence. The coding sequence is represented by a thick arrow in the macronuclear sequence. Open boxes at the ends of the phage inserts symbolize the vector arms. The positions and lengths of probes a and $\mathrm{b}$ are shown. (P) PstI. (B) Southern blot analysis of the excision of IESs 51G4404 and 51G2832 in sexual progeny of the clone transformed with $\lambda 51 \mathrm{Gmic}(\mathrm{Gmic})$. Total DNA from post-autogamous samples was digested with PstI. Because the $\mathrm{mic} / \mathrm{mac}$ ploidy ratio is $\sim 1 / 250$, only macronuclear DNA is visible. Lanes $C$ are control DNA from untransformed cells. Symbols on the right indicate the positions of IES-retaining and IES-excised macronuclear fragments. The same blot was hybridized successively with probes a and b. (C) RT-PCR analysis of $G$-gene transcripts. Total RNA samples were isolated from $\lambda 51 \mathrm{Gmic}$-transformed (Gmic) and control $(\mathrm{C})$ cells during vegetative growth $(\mathrm{V})$ and during autogamy $(\mathrm{A})$, and were then reverse-transcribed with random hexamers. Reverse transcriptase was omitted (lanes marked "-") to control for the presence of contaminating DNA. PCR amplification was performed with primers on both sides of each IES. IES-containing and IES-free PCR products (indicated by symbols on the right; 478 and 256 bp for 51G4404, 587 and 358 bp for 51G2832, and 220 and 168 bp for 51G1413, respectively) were detected on Southern blots with internal probes. Lanes $O$ and $\lambda$ are PCR controls using no DNA and $\lambda 51 \mathrm{Gmic}$ DNA, respectively.

cised (data not shown). To determine whether this difference correlates with IES transcription in the maternal macronucleus, total RNA was isolated from the transformed clone and from an untransformed control during vegetative growth and during autogamy. RNA samples were reverse-transcribed with random hexamers and PCR was performed with primers located on both sides of each IES, within flanking sequences. This allowed the simultaneous detection of transcripts from the endogenous $G$ gene, which does not contain any IES, and of IES-containing transcripts from the injected fragment. For each of the five IESs, both IES-free and IES-containing transcripts were detected during vegetative growth and autogamy of the transformed clone, as shown in Figure $2 \mathrm{C}$ for the two mcIESs and for the non-mcIES 51G1413. The incapacity of non-mcIESs to inhibit their own excision in sexual progeny is therefore not due to a lack of maternal transcription.

\section{IES inhibition is abolished in the IES ${ }^{+}$cell line upon RNAi-mediated degradation of somatic IES-containing transcripts}

To test the possibility that maternal macronuclear transcripts are nevertheless required for inhibition of mcIES excision, transcripts containing IES 51G4404 were targeted for degradation by RNAi in the $\mathrm{IES}^{+}$cell line. We used the dsRNA feeding technique (Galvani and Sperling 2002), which was shown to trigger efficient degradation of mRNA both during vegetative growth and during sexual events (Garnier et al. 2004; Nowacki et al. 2005). IES $^{+}$cells, as well as control IES ${ }^{-}$cells, were fed Escherichia coli strains producing dsRNAs homologous either to the IES sequence (ies feeding) or to the macronuclear $G$-gene sequences flanking the IES (mac feeding), both of which should target the same IES-containing transcripts. Although mac and ies dsRNA feeding did not cause visible phenotypes, a control feeding (nd7 feeding) with dsRNA homologous to ND7, a gene required for trichocyst discharge (Skouri and Cohen 1997), resulted in a mutant phenotype (nondischarge) after four to six divisions.

Autogamy was then induced by starving cells after $\sim 12$ divisions in the different feeding media. Post-autogamous cells from each culture were grown on the normal food bacterium Klebsiella, and the new macronuclear genome was probed by Southern blot analysis of total genomic DNA. Figure 3 shows that complete IES excision was observed in the sexual progeny of IES $^{+}$cells fed with mac or ies dsRNAs, but not in the progeny of cells fed with control nd7 dsRNA or with Klebsiella. An Asp718 restriction site, normally formed at the macronuclear junction by excision of IES 51G4404, could be recut in $100 \%$ of $\mathrm{IES}^{-} G$-gene copies (data not shown). Thus, RNAi-mediated targeting of IES-containing transcripts leads to complete and precise excision in the new macronuclear genome of sexual progeny, which strongly

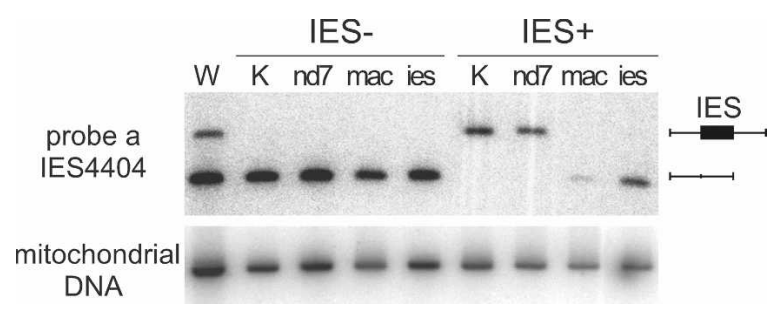

Figure 3. Excision of IES 51G4404 in the $\mathrm{IES}^{+}$cell line after RNAi treatments. Genomic DNA from the sexual progeny of cells grown before autogamy on Klebsiella $(\mathrm{K})$ or dsRNA-producing E. coli media (nd7, mac, and ies) was digested with PstI and analyzed by Southern blotting. Lane $W$ contains control DNA from IES ${ }^{-}$cells, mixed with a small amount of $\lambda 51 \mathrm{Gmic}$ prior to digestion with PstI. The same blot was hybridized with probe a (see the legend for Fig. 2) and with a probe for mitochondrial DNA as a loading control. 
suggests that maternal macronuclear transcripts are required for the excision inhibition effect.

\section{Timing of action of maternal macronuclear transcripts in IES excision inhibition}

To check the efficiency and specificity of RNAi, total RNA samples from the different feeding cultures were extracted at different time points, chosen to precede and overlap the time window during which IES excision takes place (as indicated by the detection of doublestrand breaks at IES ends by LM-PCR) (data not shown). NOWA 1/2 mRNAs, the earliest known markers of sexual events, were not detected in vegetatively growing cells but accumulated in the first autogamy time points and throughout the time course; ND7 mRNA levels were strongly reduced during vegetative growth and throughout autogamy in the nd 7 feeding culture, relative to mac or ies feeding cultures (Fig. 4A). Both sense and antisense 23-nt siRNAs accumulated during vegetative growth and autogamy but were no longer seen in vegetatively growing sexual progeny (Fig. 4B). Nd7, mac, and ies siRNAs were specifically detected in the nd7, mac, and ies feeding cultures, respectively (Supplemental Fig. S2). In all autogamous samples, 25-nt scnRNAs were detected with ies and nd7-specific probes (Supplemental Fig. S2).

We also examined the effects of dsRNA feeding on the levels of G-gene transcripts (Fig. 4C; Supplemental Fig. S3). Total RNA samples were reverse-transcribed and PCR was performed with specific primers located on both sides of IES 51G4404, within flanking sequences (Fig. 4C). In the control nd7 feeding, G-gene transcripts could still be detected during vegetative growth and autogamy (Fig. 4C, lanes 1-5), as expected. In contrast, these transcripts were degraded during vegetative growth by complementary siRNAs: Indeed, they were no longer detectable in IES ${ }^{+}$cells fed with mac or ies dsRNAs (Fig. 4C, lanes 8,13) and in IES $^{-}$cells fed with mac dsRNAs (Fig. 4C, lane 24), while IES-free G-gene transcripts could still be detected in IES- cells fed with ies siRNAs (Fig. 4C, lane 20). Surprisingly, however, in all cases, G-gene transcripts reappeared during autogamy (Fig. 4C, lanes $9,10,14-17,25,26)$ despite the continuous presence of homologous siRNAs. This stands in contrast to the continuous degradation of ND7 mRNAs by nd7 siRNAs throughout autogamy (Fig. 4A). These RNAi-resistant transcripts appear to originate from the maternal macronucleus, since they contained the IES sequence in IES ${ }^{+}$ cells, but were IES-free in IES ${ }^{-}$cells. IES-free transcripts were also detected at late stages in IES ${ }^{+}$cells fed with ies dsRNAs (Fig. 4A, lanes 16,17), indicating that the $G$ gene is transcribed in the zygotic macronucleus soon after excision of the IES. IES-free zygotic transcripts do not share homology with ies siRNAs but may be degraded by homologous mac siRNAs: Indeed, they were not detectable at the same stage in IES ${ }^{+}$cells fed with mac dsRNAs (Fig. 4A, lane 10). Thus, although RNAi targeting of IES-containing transcripts from the maternal macronucleus efficiently restores IES excision in the IES ${ }^{+}$cell line, effective degradation was observed only during vegetative growth, suggesting that the maternal transcripts that ul-
A

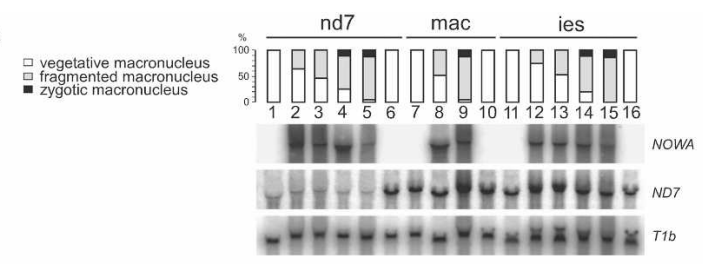

B

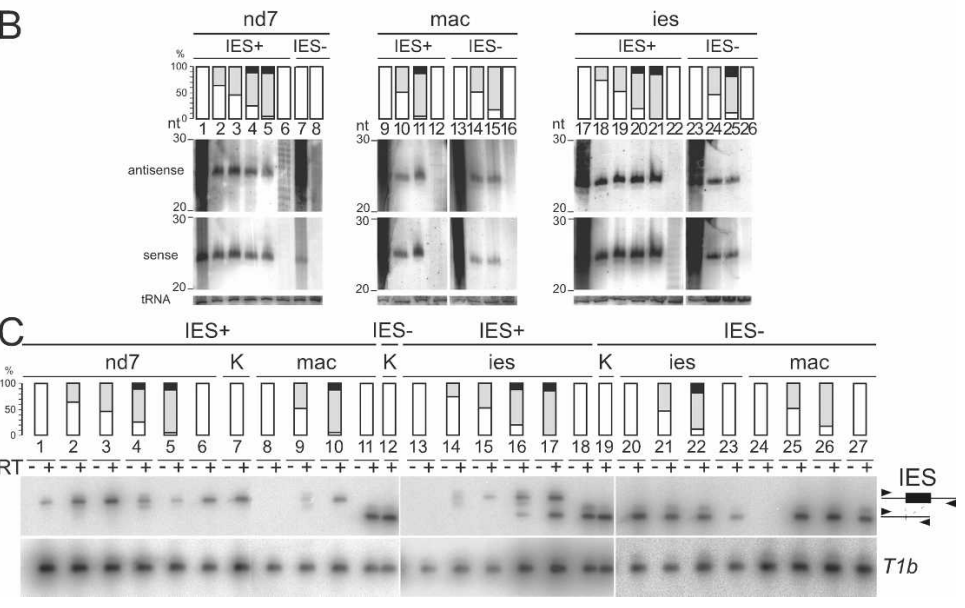

Figure 4. RNAi-mediated degradation of G-gene transcripts in the IES ${ }^{+}$and IES ${ }^{-}$cell lines. Total RNA was extracted from IES $^{-}$and IES $^{+}$cultures grown on dsRNAproducing $E$. coli media during vegetative growth and at different time points during mass autogamy or from cultures of their sexual progeny. The histograms indicate, for each time point, the percentage of cells in each cytological stage. $(A)$ Northern blot analysis of NOWA1/2 expression and ND7 silencing in the IES ${ }^{+}$ cell line. The same blot was successively hybridized with probes specific for the NOWA1/2 and ND7 mRNAs and, as a loading control, for the T1b mRNA, which encodes a component of the secretory granules (Madeddu et al. 1995). (B) Northern blot detection of 23-nt siRNAs induced by dsRNA feeding. Total RNA samples were run on a $15 \%$ polyacrylamide-urea gel, blotted, and hybridized with antisense (top panels) and sense (middle panels) oligonucleotide probes and with a 75-nt tRNA probe as a loading control (bottom panels). In vegetatively growing cultures, smears obscuring the 23-nt signal are due to the ongoing digestion of bacterial transcripts from the feeding vectors. $(C)$ RT-PCR analysis of $G$-gene transcripts at different time points after RNAi treatments. Total RNA was reverse-transcribed with random hexamers (lanes marked "-" are controls without reverse transcriptase), and PCR amplification was performed with primers on both sides of IES 51G4404, outside the fragment used for mac feeding. PCR products from IES-containing $(832 \mathrm{bp})$ and IES-free $(610 \mathrm{bp})$ are indicated at the right of the top panel. Total RNA was extracted from IES ${ }^{+}$and IES ${ }^{-}$vegetative cultures grown on Klebsiella (K). (Bottom panel) As a control for RT, specific primers on both sides of a 28 -nt intron of the T1b gene were used for PCR amplification. 
timately result in excision inhibition are carried over from vegetative growth or early meiotic stages, or exert their action during these early steps. The latter possibility is consistent with their presumed role in sequestering or degrading the homologous scnRNAs produced by meiotic micronuclei.

\section{Microinjection of 23-nt and 25-nt RNA duplexes into conjugating IES ${ }^{+}$cells triggers excision at different stages}

The 23-nt siRNAs produced by dsRNA feeding may trigger IES excision by degrading early maternal macronuclear transcripts, thereby freeing complementary scnRNAs to target the IES in the developing macronucleus. An alternative possibility is that the 23-nt siRNAs, which persist in the cells throughout autogamy, could themselves directly target IES excision in the developing macronucleus. However, while such a direct role may explain the effect of siRNAs matching the IES sequence, it cannot easily account for the effect of siRNAs from flanking sequences, which is therefore best explained by the degradation of maternal transcripts. To gain further insight into the respective roles of siRNAs and scnRNAs, we tested the capacity of 23-nt and 25-nt synthetic RNA duplexes to promote excision in the IES ${ }^{+}$ cell line. Both types of duplexes matched the same internal region of IES 51G4404 and were designed to form the typical 2-nt $3^{\prime}$ overhangs resulting from RNase III cleavage, which was shown to be the structure of scnRNA duplexes (G. Lepère, M. Nowacki, V. Serrano, J.-F. Gout, S. Duharcourt, and E. Meyer, in prep.). Synthetic RNA duplexes were microinjected into the cytoplasm of synchronized conjugating IES ${ }^{+}$cells at different times: during meiosis of the micronuclei (starting $3 \mathrm{~h}$ after the mixing of mating types), during mitotic divisions of the zygotic nucleus (4.5-6.5 h) (Grandchamp and Beisson 1981), and during development of new macronuclei, up to the peak of IES excision events $(6.5-11 \mathrm{~h})$ (Fig. 5; Betermier et al. 2000). The progeny of individual injected cells were grown for $\sim 12$ divisions, and total DNA was extracted and submitted to a PCR assay, yielding 501-bp and 279-bp products from IES-retaining and IESexcised forms of the $G$ gene, respectively (Fig. 5).

Table 1 summarizes the results obtained in two separate experiments after injection of the 23-nt and 25-nt synthetic RNA duplexes. Twenty-four percent (22 out of 90) of cells injected with the 23-nt synthetic RNA duplex gave rise to progeny showing some excision of the IES in the new macronucleus. In contrast, only $4 \%$ (two out of 54) of the progeny of cells injected with a control synthetic RNA duplex homologous to the ND7 gene showed some IES excision, which represents the rate of spontaneous reversion to excision of conjugating IES $^{+}$cells under the experimental conditions used. Figure $5 \mathrm{~A}$ shows the results obtained for 24 representative clones after injection of the 23-nt synthetic IES duplex at different times. In most of the clones showing detectable excision, the effect was only partial and the new macronucleus still contained a large fraction of $G$-gene copies retaining

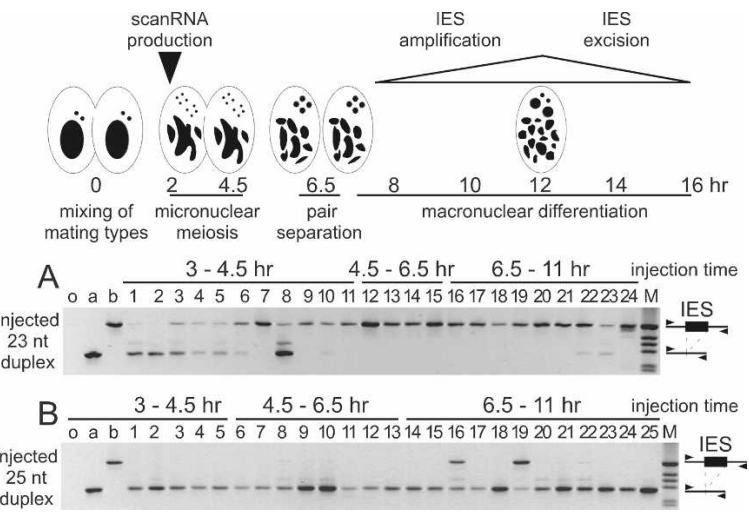

Figure 5. Microinjection of synthetic short RNA duplexes restores IES excision. The drawings at the top show a schematic representation of the timing of nuclear and molecular events associated with conjugation. The events start with mixing cells of complementary mating types, and the approximate time periods for several of these are indicated. Above is shown that scnRNAs are produced during micronuclear meiosis (G. Lepère, M. Nowacki, V. Serrano, J.-F. Gout, S. Duharcourt, and E. Meyer, in prep.) and the period when IES excision within the zygotic macronucleus takes place (Betermier et al. 2000). Progressive degradation of the maternal macronucleus starts shortly after meiosis of the micronuclei and the resulting fragments persist within the cytoplasm throughout the whole period of formation of the new zygotic macronucleus. Twentythree-nucleotide $(A)$ and 25-nt $(B)$ synthetic RNA duplexes with 2-nt 3' overhangs, both matching the same internal sequence of IES 51G4404, were microinjected into IES ${ }^{+}$mating cells at different times after the mixing of mating types, as indicated above. IES-containing and IES-excised macronuclear $G$-gene copies were amplified by the same PCR from total DNA extracted from cultured individual progeny of injected mating pairs (lanes 1-24 for $A$, and lanes 1-25 for $B$ ), from IES ${ }^{-}$cells (lane $a$ ), and from the progeny of an uninjected pair (lane $b$ ). Lane $o$ is a negative control with no DNA. Symbols on the right indicate the positions of IES-containing (501 bp) and IES-free (279 bp) PCR products. PCR products were visualized by ethidium bromide staining. The size marker $(\mathrm{M})$ in the right-most lane is a $1-\mathrm{kb}$ DNA ladder (Invitrogen).

the IES. Interestingly, the effect strongly depended on the time of injection. Mating cells injected during meiosis gave a higher fraction of IES-excised progeny $162.5 \%$, 15 out of 24) than those injected at later time points $(13 \%$, seven out of 54$)$. Thus, the $23-n t$ synthetic IES duplex can lead to complete excision when injected during micronuclear meiosis, but has little or no effect when injected at times closer to the period of IES excision, suggesting that 23-nt siRNAs trigger excision in an indirect manner.

Injection of the 25-nt synthetic IES duplex gave different results. A much larger fraction of injected cells gave rise to progeny showing excision in the new macronucleus $(75 \%, 34$ out of 45$)$ (Table 1). The difference was particularly striking when duplexes were injected at middle to late stages $(4.5-11 \mathrm{~h})$ : Seventy-two percent $(28$ out of 39 ) of progeny showed excision, versus $13 \%$ (seven out of 54) for the 23-nt synthetic duplex. Furthermore, in almost all cases, excision induced by the 25 -nt synthetic 
Table 1. Frequencies of IES excision after microinjection of short synthetic RNA duplexes into conjugating IES ${ }^{+}$cells

\begin{tabular}{lclrlcl}
\hline $\begin{array}{l}\text { Injection } \\
\text { time } \\
(\mathrm{hr})^{\mathrm{b}}\end{array}$ & \multicolumn{5}{c}{ IES excision $^{\mathrm{a}}$} \\
\cline { 2 - 7 } & Control duplex $^{\mathrm{c}}$ & \multicolumn{2}{c}{ 23-nt duplex } & 25-nt duplex \\
\hline $3-4.5$ & $1 / 8$ & 0.12 & $15 / 24$ & 0.625 & $6 / 6$ & 1 \\
$4.5-6.5$ & $0 / 12$ & 0 & $2 / 19$ & 0.10 & $11 / 14$ & 0.78 \\
$6.5-11$ & $1 / 34$ & 0.03 & $5 / 35$ & 0.14 & $17 / 25$ & 0.68 \\
Total & $2 / 54$ & 0.04 & $22 / 90$ & 0.24 & $34 / 45$ & 0.75 \\
\hline
\end{tabular}

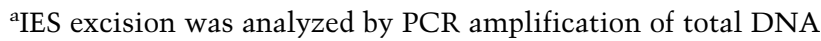
extracted from progeny of individual injected cells. The table gives the numbers and frequencies of injected cells giving rise to progeny showing detectable excision for each injection experiment.

${ }^{\mathrm{b}}$ Sexually reactive $\mathrm{IES}^{+}$cells of complementary mating types were mixed at $t=0$ and conjugating cells or exconjugants were injected at the indicated times.

${ }^{\mathrm{c}}$ The 23-nt and 25-nt synthetic RNA duplexes matched the same internal sequence in IES 51G4404. The control duplex is a 25-nt synthetic duplex matching the unrelated ND7 gene.

duplex was complete and occurred in 100\% of G-gene copies in the new macronucleus, as shown in Figure 5B for a representative sample of 25 clones. Thus, in contrast to the 23-nt synthetic duplex, the 25-nt synthetic duplex efficiently restores IES excision in the IES $^{+}$cell line whether it is injected before or during the period of IES excision, suggesting that 25-nt scnRNAs target IES excision in a more direct manner. In all cases, the 279-bp PCR fragment could be recut by Asp718 (data not shown), demonstrating that IES excision triggered by both types of RNA duplexes was precise at the nucleotide level.

\section{Discussion}

Evidence for noncoding transcripts of the maternal macronuclear genome during vegetative growth and sexual events

We identified low-abundance transcripts of the $G$ surface antigen gene that can be detected by RT-PCR both during vegetative growth and during sexual events. These transcripts appear to be non-protein-coding RNAs, because (1) Northern blot analyses indicated that the cell cultures used were expressing the $A$ surface antigen, and only one surface antigen mRNA is normally found in vegetative clones as a consequence of the mutually exclusive expression of these genes (Caron and Meyer 1989), and (2) both strands of the G-gene coding sequence are transcribed at similar levels, as shown by strand-specific RT-PCR (Fig. 1). We show that G-gene transcripts from the IES ${ }^{+}$cell line contain IES 51G4404, while those from the IES ${ }^{-}$cell line do not. Since these two lines have the same micronuclear genome and differ only by the presence or absence of the IES in the macronuclear genome, we conclude that the transcripts originate from the macronucleus. When the micronuclear version of the $G$ gene is introduced into the macronucleus, transcripts can be detected for each of the five IESs in the coding sequence (Fig. 2). Low-level noncoding transcripts are not limited to the $G$ gene, since RT-PCR products can also be amplified from several intergenic regions of the macronuclear genome, including the region upstream of the transcription start site of the $A$ gene (data not shown). Furthermore, it was shown previously that even a bacterial plasmid can be transcribed when introduced into the macronucleus (Galvani and Sperling 2001), suggesting a promiscuous transcription that does not require specific promoter sequences.

IES excision is restored in the progeny of IES $S^{+}$cells upon RNAi-mediated degradation of somatic IES-retaining transcripts

Using the feeding technique for RNAi, we show that targeting the macronuclear transcripts containing IES $51 \mathrm{G} 4404$ in the $\mathrm{IES}^{+}$cell line results in the complete and precise excision of the IES in the new somatic genome of sexual progeny. In this technique, the bacterial dsRNAs ingested by Paramecium cells are processed into 23-nt siRNAs that target the post-transcriptional degradation of homologous RNAs (Galvani and Sperling 2002; Garnier et al. 2004; Nowacki et al. 2005). Although we cannot formally exclude the possibility that 23-nt siRNAs matching the IES directly target excision in the zygotic macronucleus, our results fully support an alternative explanation. Importantly, efficient IES excision was induced not only by dsRNAs corresponding to the sequence of the IES itself but also by dsRNAs containing only its flanking coding sequences, while dsRNAs from the unrelated ND7 gene had no effect. Northern blot analyses showed that the processing of flanking-sequence dsRNAs did not result in detectable production of siRNAs outside the fragment used for dsRNA production-i.e., from the IES sequence-or in any obvious change in the amount of IES-specific scnRNAs (Supplemental Fig. S2).

Since flanking-sequence siRNAs cannot trigger excision of the zygotic IES through a direct recognition of the IES sequence, their effect is likely the result of degradation of macronuclear transcripts containing the IES and flanking sequences. These transcripts, which are efficiently degraded during vegetative growth by both types of $G$-gene siRNAs (Fig. 4C), thus appear to be required to mediate the inhibitory effect of the maternal macronucleus in the $\mathrm{IES}^{+}$cell line.

\section{IES-containing transcripts from the maternal macronuclear genome likely act during meiosis to inhibit IES excision later in development}

RT-PCR analyses of samples taken at different time points during mass autogamies revealed that siRNA-mediated degradation of maternal noncoding transcripts is effective only during vegetative growth, as RNAi-resistant transcripts reappeared during autogamy in the IES ${ }^{+}$ and IES ${ }^{-}$cell lines. This is clearly in contrast with the 
continuous depletion of ND7 mRNAs during autogamy (Fig. 4A), and with the previously reported efficiency of the feeding technique in degrading mRNAs from the NOWA1 gene, which is specifically expressed during sexual events (Nowacki et al. 2005). Developmental regulation of the cellular localization of the noncoding RNAs and/or their association with specific RNA-binding proteins may explain how those RNAs produced during autogamy could escape degradation by the RNAi machinery.

RNAi-resistant transcripts that reappear during autogamy of the IES ${ }^{+}$cell line are unable to mediate excision inhibition. This may be because they are produced too late to completely sequester or degrade IES-specific scnRNAs before zygotic macronuclei are formed. That the maternal IES-containing transcripts need to be present at early stages is also supported by the effects of 23-nt synthetic siRNAs matching the IES sequence, which can induce IES excision only if they are microinjected during meiosis into conjugating IES $^{+}$cells; i.e., $\sim 7$ $\mathrm{h}$ before excision actually occurs, but not later. Similarly, the production of 23- to 24-nt siRNAs from a hairpin construct driven by an inducible promoter in $T$. thermophila was shown to target elimination of the homologous locus during macronuclear development; induction was much more efficient at the earliest stages of conjugation, well before genome rearrangements, suggesting that a similar indirect mechanism may be at work (Howard-Till and Yao 2006). The requirement of IES-containing maternal macronuclear transcripts at early stages does not support the idea that they could inhibit excision by directly pairing with the IES sequence in the developing macronucleus, and fits better a role in the scanning mechanism, which could be to filter the pool of scnRNAs produced by the micronucleus during meiosis (Fig. 6).

\section{Direct role of scnRNAs in IES excision}

The scanning model assumes that the molecules that ultimately target DNA elimination in the developing macronucleus are the meiosis-specific scnRNAs. This model was initially based on indirect evidence established in T. thermophila: The knockout of Dicer-like (DCL1) or Argonaute/Piwi (TWI1) genes was shown to cause defects both in the accumulation of 27- to 30-nt scnRNAs and in DNA elimination during macronuclear development (Mochizuki et al. 2002; Mochizuki and Gorovsky 2004a, 2005; Malone et al. 2005). In P. tetraurelia, the 25-nt scnRNAs are likely to be functionally equivalent to the T. thermophila 27- to 30-nt scnRNAs (G. Lepère, M. Nowacki, V. Serrano, J.-F. Gout, S. Duharcourt, and E. Meyer, in prep.). The microinjection into conjugating IES $^{+}$cells of a 25 -nt synthetic RNA duplex matching the IES sequence, with 2-nt 3' overhangs mimicking the structure of scnRNA duplexes, provides direct evidence for the capacity of scnRNAs to target deletions. Indeed, the 25-nt synthetic duplex is not only more efficient in triggering IES excision than the 23-nt synthetic duplex matching the same sequence, but also,

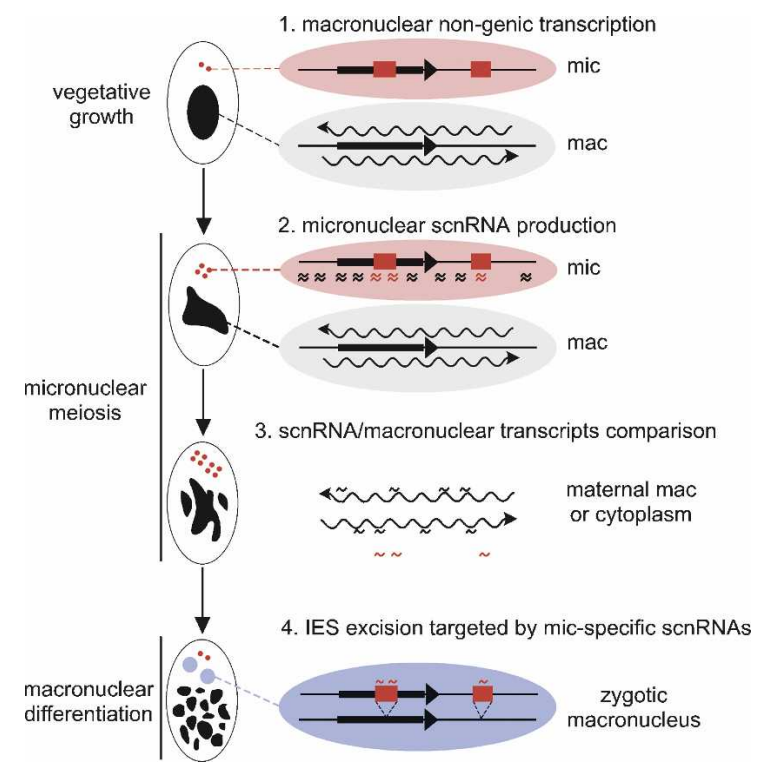

Figure 6. The maternal scnRNA/macronuclear RNA scanning model. (1) During vegetative growth, nongenic transcription of all or most of the macronuclear genome occurs on both strands. (2) Upon initiating meiosis, short double-stranded scnRNAs from the micronuclear genome are produced by an RNAi-dependent pathway. (3) ScnRNAs scan the maternal macronuclear genome by base-pairing with noncoding macronuclear transcripts either in the maternal macronucleus or in the cytoplasm, during meiosis or soon after. (4) ScnRNAs that pair with homologous macronuclear transcripts are sequestered or destroyed, while micronuclear-specific scnRNAs pair with homologous nascent transcripts in the zygotic macronucleus and target IES excision.

and more importantly, is able to do so at all stages tested. At early meiotic stages, the large number of molecules injected can be expected to override the inhibitory effect of the low-abundance, IES-containing transcripts from the maternal macronucleus. When injected at later stages, after the presumed period of scanning of maternal transcripts and closer to the actual time of IES excision, the fact that the 25-nt synthetic duplex can still promote excision, whereas the 23-nt synthetic duplex cannot, strongly suggests that scnRNAs directly recognize germline-specific sequences in the developing macronucleus.

The exact mechanisms of this recognition and of the recruitment of the excision machinery are not yet clear. Base-pairing could occur between scnRNAs and target DNA, or between scnRNAs and nascent transcripts, as has been proposed to explain the targeting of heterochromatin formation in Schizosaccharomyces pombe (Buhler et al. 2006). It is tempting to imagine that similar pairing interactions between scnRNAs and nascent transcripts occur first in the maternal macronucleus, leading to the sequestration or degradation of paired scnRNAs, and later in the developing macronucleus, but with a different outcome: Paired scnRNAs would then be a signal for epigenetic modifications of germline-limited sequences. In Tetrahymena, scnRNAs appear to direct chromatin modifications on homologous germline se- 
quences during macronuclear development (Taverna et al. 2002; Liu et al. 2004, 2007; Malone et al. 2005; Mochizuki and Gorovsky 2005). Paramecium scnRNAs may operate through a similar mechanism, although the short lengths of most IESs, which cannot accommodate even a single nucleosome, would suggest more precisely localized modifications, such as the methylation or other modification of specific nucleotides.

\section{The maternal scnRNA/macronuclear RNA scanning model}

Taken together, the results presented here support a modified version of the scanning model, in which the scnRNAs would scan the maternal macronuclear genome by base-pairing with noncoding macronuclear transcripts. For each genomic sequence, the outcome of scanning would thus depend on the relative cellular concentration of two types of maternal RNA molecules with antagonistic functions. In the $\mathrm{IES}^{+}$cell line, scnRNAs matching the IES would be titrated by the macronucleusderived transcripts containing the IES and would thus no longer be available to target IES excision. We have shown previously that, for a given IES copy number in the maternal macronucleus, the probability of inhibition varies greatly between different IESs, suggesting differences in some intrinsic properties of these elements (Duharcourt et al. 1998). The maternal transcription level and/or the stability of IES-containing transcripts may, at least in part, determine the characteristic inhibition efficiency of each IES. The recognition of non-mc-IESs may not depend on scnRNAs or may involve other RNA molecules that are not selected by scanning maternal macronuclear transcripts. Indeed, we showed that transcription of nonmc-IESs introduced into the maternal macronucleus does not inhibit excision in the developing macronucleus.

Whether the scanning process occurs in the cytoplasm or in the macronucleus is unknown. It may be that the macronucleus-derived transcripts never leave the macronucleus and thus serve as a molecular sponge, absorbing the scnRNAs with which they interact /Chalker et al. 2005). In Tetrahymena, the localization of the scnRNA-associated protein Twilp, first in the maternal macronucleus and then in the developing macronucleus, has led to the proposal that scanning occurs within the maternal macronucleus and that the selected scnRNAs are then actively transported to the zygotic macronucleus (Mochizuki and Gorovsky 2004b). The Paramecium RNA-binding NOWA1/2 proteins, which are required for the excision of maternally regulated IESs, are massively transferred from the maternal to the zygotic macronucleus and are possibly involved both in the pairing and in the transport of scnRNAs (Nowacki et al. 2005).

The basic mechanism of the scnRNA/macronuclear RNA scanning model may account for all known epigenetic effects on genome rearrangements in Paramecium and Tetrahymena, including the maternal inheritance of macronuclear gene deletions and the induction of such deletions by siRNAs (Garnier et al. 2004). In essence, this mechanism is similar to the "target mimicry" mechanism described in Arabidopsis thaliana, in which a noncoding transcript with imperfect complementarity sequesters one miRNA away from its more complementary target mRNA (Franco-Zorrilla et al. 2007). In the two ciliates, however, genome-wide RNA mimics (the maternal macronuclear transcripts) are identical in sequence to the targets (zygotic transcripts) but are produced at an earlier developmental stage and in a different nucleus. The temporal and spatial distribution of mimics and targets would be at the basis of the scnRNAmediated natural genomic subtraction. Maternal macronuclear transcripts also appear to regulate developmental genome rearrangements in the very distant ciliate Oxytricha trifallax, but in a fundamentally different manner, acting as templates that guide the developmental "unscrambling" of micronuclear genes (Nowacki et al. 2008). Unscrambling is not known to occur in Paramecium or Tetrahymena, and in the case of maternal inhibition of IES excision, there is experimental evidence in Paramecium that maternal transcripts do not act as templates guiding excision or local synthesis of DNA (Duharcourt et al. 1995). The present results highlight the diversity of homology-dependent mechanisms through which maternal noncoding transcripts regulate zygotic genome rearrangements in ciliates and may perhaps mediate epigenetic regulation of the zygotic genome in other eukaryotes.

\section{Materials and methods}

Paramecium strains, cultivation, and autogamy

All experiments were carried out with the entirely homozygous strain 51 . The IES ${ }^{+}$cell line was obtained after transformation of vegetative macronucleus with plasmid p851 (Duharcourt et al. 1995) and selection of the sexual progenies in which IES 51 G4404 was present in 100\% of the macronuclear chromosomes. Cells were grown in wheat grass powder (WGP) (Pines International) infusion medium bacterized the day before use with Klebsiella pneumoniae, unless otherwise stated, and supplemented with $0.8 \mathrm{mg} / \mathrm{mL} \beta$-sitosterol (Merck). Cultivation and autogamy were carried out at $27^{\circ} \mathrm{C}$ as described previously (Duharcourt et al. 1998).

\section{Feeding experiments}

The plasmids used in the feeding experiments were obtained by subcloning the PstI-SacI restriction fragment from plasmid p629 for the mac feeding and HindIII-Asp718 restriction fragment from plasmid pIES for the ies feeding (Duharcourt et al. $1995)$ into the feeding vector and E. coli strain described in Galvani and Sperling (2002). The nd7 feeding vector was the same as in Garnier et al. (2004).

Feeding media were prepared by inoculating precultures of the appropriate feeding strains into WGP Paramecium medium containing ampicillin at $0.1 \mathrm{mg} / \mathrm{mL}$ and growing them overnight with shaking at $37^{\circ} \mathrm{C}$. On the next day, the culture was diluted fourfold into the same medium. After $30 \mathrm{~min}$ at $37^{\circ} \mathrm{C}$, IPTG was added at a final concentration of $0.4 \mathrm{mM}$ to induce the synthesis of dsRNA, and the culture was incubated for $3 \mathrm{~h}$ 
at $37^{\circ} \mathrm{C}$. The medium was cooled down at $27^{\circ} \mathrm{C}$ and supplemented with $0.8 \mathrm{mg} / \mathrm{mL} \beta$-sitosterol just before use.

\section{Microinjections}

For DNA injection, Paramecium cells from a single caryonidal clone were microinjected in Volvic mineral water (Volvic), containing $0.2 \%$ bovine serum albumin (BSA), under an oil film (Nujol), while they were visualized in a phase-contrast inverted microscope (Axiovert 35M, Zeiss). CsCl-purified $\lambda$ phage DNA was cut on either side of the insert for $\lambda \mathrm{Gmic}$, and restriction digests were phenol-extracted, filtered on 0.22 - $\mu \mathrm{m}$ pore-size U1trafree-MC filter (Millipore), and precipitated with ethanol. Approximately $5 \mathrm{pL}$ of a $5 \mathrm{mg} / \mathrm{mL}$ solution in water was delivered into the macronucleus. Injected DNA molecules replicate autonomously at stable copy numbers in the vegetative macronucleus.

RNA injection into conjugating cells was performed following the method for DNA injection. Conjugation was initiated by mixing sexually reactive cells from complementary mating types. At $2 \mathrm{~h}$ after mixing, pairs firmly engaged in conjugation were manually transferred to depressions filled with exhausted medium. Pairs separated at $6 \mathrm{~h} 30 \mathrm{~min}$ after mixing. We typically injected into the cytoplasm of one cell in each pair. The injected pairs or exconjugants were transferred to individual depressions filled with exhausted medium for $24 \mathrm{~h}$, and were then grown in normal Klebsiella medium for $3 \mathrm{~d}$ before a small amount of DNA was extracted using the Nucleospin kit (Macherey-Nagel). DNA was analyzed by PCR to assay the presence of the IES in the macronuclear genome. The viability of injected pairs with 23- or 25-nt synthetic RNA duplexes matching the same internal sequence in IES 51G4404 was the same as that of uninjected pairs or pairs injected with the control duplex matching the unrelated ND7 gene.

RNA duplexes were formed by mixing equal amounts of the complementary oligonucleotides; the denaturating and cooling steps were $5 \mathrm{~min}$ at $95^{\circ} \mathrm{C}, 10 \mathrm{sec}$ at $70^{\circ} \mathrm{C}$, and a slow decrease to $4^{\circ} \mathrm{C}$ at a rate of $1^{\circ} \mathrm{C}$ per minute. The final RNA concentrations were $80 \mathrm{pmol} / \mu \mathrm{L}$. The sequences of the synthetic RNA duplexes injected were the following: 23-nt IES 51G4404, 5'P-UU CAAGAAUUAGAAAUCUAUUCA-3' $/ 5^{\prime}$ P-AAUAGAUUUCT AAUUCUUGAAAU-3'; 25-nt IES 51G4404， 5'P-UUUCAA GAAUUAGAAAUCUAUUCAG-3'/5'P-GAAUAGAUUUCUA AUUCUUGAAAUA-3'; 25-nt ND7, 5'P-UUGAUGAAUUCG CAAAGAAUAAAGA-3' ${ }^{\prime} / 5^{\prime}$ P-UUUAUUCUUUGCGAAUUCA UCAAUA - $3^{\prime}$

\section{DNA and RNA extraction}

One-hundred-milliliter to 400-mL cultures of exponentially growing cells at 1000 cells per milliliter or of autogamous cells at 3000 cells per milliliter were centrifuged. For DNA extraction, cell pellets were washed in $10 \mathrm{mM}$ Tris- $\mathrm{HCl}(\mathrm{pH} 7.0)$, resuspended in $1 \mathrm{vol}$ of the same buffer, and quickly added to 4 vol of lysis solution (0.44 M EDTA at pH 9.0, 1\% SDS, 0.5\% $\mathrm{N}$-laurylsarcosine [Sigma], $1 \mathrm{mg} / \mathrm{mL}$ proteinase $\mathrm{K}$ [Merck]). The lysates were incubated overnight at $55^{\circ} \mathrm{C}$, gently extracted with phenol, and dialyzed twice against TE $(10 \mathrm{mM}$ Tris- $\mathrm{HCl}, 1 \mathrm{mM}$ EDTA at $\mathrm{pH} 8.0$ ) containing $20 \%$ ethanol and once against TE. RNA was extracted from unwashed cell pellets with the TRIzol (Invitrogen) procedure, modified by the addition of glass beads.

\section{Southern and Northern blots}

DNA and RNA electrophoreses were carried out according to standard procedures (Sambrook and Russell 2001). Genomic
DNA (1-2 $\mu \mathrm{g})$, or RT-PCR products were transferred from agarose gels to Hybond $\mathrm{N}^{+}$membranes (Amersham) in $0.4 \mathrm{~N} \mathrm{NaOH}$ after depurination in $0.25 \mathrm{~N} \mathrm{HCl}$. For agarose Northern, total RNA $(20 \mu \mathrm{g})$ was separated on $1 \%$ agarose gel in Tris Borate EDTA, transferred to Hybond $\mathrm{N}^{+}$membrane in $20 \times$ SSC buffer, and UV cross-linked. For short RNA Northerns, total RNA (20 $\mu \mathrm{g}$ ) was separated on $15 \%$ polyacrylamide-urea gels (Long Ranger, BioWhittaker Molecular Applications), transferred to Hybond $\mathrm{N}^{+}$under vacuum in $20 \times$ SSC, and UV cross-linked. Hybridization was carried out in $7 \%$ SDS, $0.5 \mathrm{M}$ sodium phosphate, $1 \% \mathrm{BSA}$, and $1 \mathrm{mM}$ EDTA $(\mathrm{pH} 7.2)$ at $60^{\circ} \mathrm{C}$ decreasing to $30^{\circ} \mathrm{C}$ for oligonucleotide probes.

Double-stranded probes were labeled by random priming with $\left[\alpha-{ }^{32} \mathrm{P}\right] \mathrm{dATP}\left(3000 \mathrm{Ci} / \mathrm{mmol}^{\prime}\right.$ Amersham $)$. Oligonucleotide probes were labeled with $\left[\gamma^{32} \mathrm{P}\right] \mathrm{ATP}(5000 \mathrm{Ci} / \mathrm{mmol}$; Amersham) and T4 polynucleotide kinase. Membranes were then washed for at least $30 \mathrm{~min}$ in $0.2 \times \mathrm{SSC}, 0.1 \%$ SDS at $55^{\circ} \mathrm{C}-60^{\circ} \mathrm{C}$ (or $2 \times$ SSC, $0.1 \%$ SDS at room temperature for short RNA Northern blots) prior to image plate exposure.

The NOWA $1 / 2$ probe corresponds to an $856-$ bp PCR fragment covering nucleotides 2401-3256 of accession number AJ876761; the ND7 probe corresponds to an 873-bp fragment covering nucleotides 461-1333 of accession number Y07803; and the $T 1 b$ probe corresponds to a 258-bp fragment covering nucleotides 892-1149 of accession number U47115. The tRNA and mitochondrial DNA probes are available upon request.

\section{$R T-P C R$}

Before RT reactions, total RNA (4 $\mu$ g) was treated with DNaseI (Fermentas) either for $2 \mathrm{~h}$ when RNA was reverse-transcribed with random hexamers or overnight at $37^{\circ} \mathrm{C}$ for gene-specific reverse transcription. DNaseI was inactivated by addition of EDTA to $2.5 \mathrm{mM}$ and incubation for $10 \mathrm{~min}$ at $65^{\circ} \mathrm{C}$. Of the treated RNA, $0.5 \mu \mathrm{g}$ was reversed-transcribed using random hexamers (225 ng) with SuperScript II reverse transcriptase (Invitrogen) according to the supplier's instructions. For strandspecific RT-PCR, a linker sequence, LK 5'-CGACTGGAGCA CGAGGACACTGA-3', was attached to the 5' end of a primer specific for each strand (Cho et al. 2005), and 2 pmol of this chimeric oligonucleotide was used to prime RT of $1 \mu \mathrm{g}$ of the treated RNA. One-twentieth of these reactions was used as a template for PCR amplification. PCR amplifications were performed in $25 \mu \mathrm{L}$ of $1 \times$ DyNAzyme buffer, with $10 \mathrm{pmol}$ of each primer, $5 \mathrm{nmol}$ of each deoxynucleoside triphosphate (dNTP), and $1 \mathrm{U}$ of DyNAzyme II DNA polymerase (Finnzymes). The standard program was run on an Eppendorf thermal cycler: 2 min at $94^{\circ} \mathrm{C}, 30$ cycles of $30 \mathrm{sec}$ at $94^{\circ} \mathrm{C}, 30 \mathrm{sec}$ at the appropriate annealing temperature, and $1 \mathrm{~min}$ at $72^{\circ} \mathrm{C}$, followed by a final step of $5 \mathrm{~min}$ at $72^{\circ} \mathrm{C}$. In strand-specific RT-PCR, the LK sequence alone was used as a primer with a gene-specific primer for PCR reactions. The sequences of oligonucleotides used for RT and PCR are available upon request. RT-PCR products were cloned into the TOPO-TA vector (Invitrogen).

\section{Acknowledgments}

We thank all members of the laboratory for fruitful discussions and Allison Mallory for critical reading of the manuscript. G.L. was the recipient of fellowships from the Ministère de l'Education Nationale and the Association pour la Recherche sur le Cancer. Work in our laboratory was supported by the CNRS, the Ministère de $1^{\prime}$ Education Nationale, de la Recherche et de la Technologie (ACI BCMS 2004 \#BCMS287), the Association pour la Recherche sur le Cancer (grant \#3608), the 
Comité de Paris de la Ligue Nationale contre le Cancer (grant \#RS06/75-32), and contract NT05-2_41522 from the Agence Nationale de la Recherche.

\section{References}

Aury, J.M., Jaillon, O., Duret, L., Noel, B., Jubin, C., Porcel, B.M., Segurens, B., Daubin, V., Anthouard, V., Aiach, N., et al. 2006. Global trends of whole-genome duplications revealed by the ciliate Paramecium tetraurelia. Nature 444: 171-178.

Berger, J.D. 1973. Nuclear differentiation and nucleic acid synthesis in well-fed exconjugants of Paramecium aurelia. Chromosoma 42: 247-268.

Berger, J.D. 1986. Autogamy in Paramecium. Cell cycle stagespecific commitment to meiosis. Exp. Cell Res. 166: 475485.

Betermier, M., Duharcourt, S., Seitz, H., and Meyer, E. 2000. Timing of developmentally programmed excision and circularization of Paramecium internal eliminated sequences. Mol. Cell. Biol. 20: 1553-1561.

Buhler, M., Verdel, A., and Moazed, D. 2006. Tethering RITS to a nascent transcript initiates RNAi- and heterochromatindependent gene silencing. Cell 125: 873-886.

Caron, F. and Meyer, E. 1989. Molecular basis of surface antigen variation in paramecia. Annu. Rev. Microbiol. 43: 23-42.

Chalker, D.L. and Yao, M.C. 1996. Non-Mendelian, heritable blocks to DNA rearrangement are induced by loading the somatic nucleus of Tetrahymena thermophila with germ line-limited DNA. Mol. Cell. Biol. 16: 3658-3667.

Chalker, D.L., Fuller, P., and Yao, M.C. 2005. Communication between parental and developing genomes during Tetrahymena nuclear differentiation is likely mediated by homologous RNAs. Genetics 169: 149-160.

Cho, D.H., Thienes, C.P., Mahoney, S.E., Analau, E., Filippova, G.N., and Tapscott, S.J. 2005. Antisense transcription and heterochromatin at the DM1 CTG repeats are constrained by CTCF. Mol. Cell 20: 483-489.

Duharcourt, S., Butler, A., and Meyer, E. 1995. Epigenetic selfregulation of developmental excision of an internal eliminated sequence on Paramecium tetraurelia. Genes \& Dev. 9: 2065-2077.

Duharcourt, S., Keller, A.M., and Meyer, E. 1998. Homologydependent maternal inhibition of developmental excision of internal eliminated sequences in Paramecium tetraurelia. Mol. Cell. Biol. 18: 7075-7085.

Franco-Zorrilla, J.M., Valli, A., Todesco, M., Mateos, I., Puga, M.I., Rubio-Somoza, I., Leyva, A., Weigel, D., Garcia, J.A., and Paz-Ares, J. 2007. Target mimicry provides a new mechanism for regulation of microRNA activity. Nat. Genet. 39: 1033-1037.

Galvani, A. and Sperling, L. 2001. Transgene-mediated posttranscriptional gene silencing is inhibited by 3 ' non-coding sequences in Paramecium. Nucleic Acids Res. 29: 4387-4394. doi: 10.1093/nar/29.21.4387.

Galvani, A. and Sperling, L. 2002. RNA interference by feeding in Paramecium. Trends Genet. 18: 11-12.

Garnier, O., Serrano, V., Duharcourt, S., and Meyer, E. 2004. RNA-mediated programming of developmental genome rearrangements in Paramecium tetraurelia. Mol. Cell. Biol. 24: 7370-7379.

Grandchamp, S. and Beisson, J. 1981. Positional control of nuclear differentiation in Paramecium. Dev. Biol. 81: 336341.

Gratias, A. and Betermier, M. 2003. Processing of double-strand breaks is involved in the precise excision of Paramecium internal eliminated sequences. Mol. Cell. Biol. 23: 7152-7162.

Howard-Till, R.A. and Yao, M.C. 2006. Induction of gene silencing by hairpin RNA expression in Tetrahymena thermophila reveals a second small RNA pathway. Mol. Cell. Biol. 26: 8731-8742.

Klobutcher, L.A. and Herrick, G. 1995. Consensus inverted terminal repeat sequence of Paramecium IESs: Resemblance to termini of Tc1-related and Euplotes Tec transposons. Nucleic Acids Res. 23: 2006-2013. doi: 10.1093/nar/ 23.11.2006.

Klobutcher, L.A. and Herrick, G. 1997. Developmental genome reorganization in ciliated protozoa: The transposon link. Prog. Nucleic Acid Res. Mol. Biol. 56: 1-62.

Kowalczyk, C.A., Anderson, A.M., Arce-Larreta, M., and Chalker, D.L. 2006. The germ line limited M element of Tetrahymena is targeted for elimination from the somatic genome by a homology-dependent mechanism. Nucleic Acids Res. 34: 5778-5789. doi: 10.1093/nar/gk1699.

Le Mouel, A., Butler, A., Caron, F., and Meyer, E. 2003. Developmentally regulated chromosome fragmentation linked to imprecise elimination of repeated sequences in paramecia. Eukaryot. Cell 2: 1076-1090.

Lee, S.R. and Collins, K. 2006. Two classes of endogenous small RNAs in Tetrahymena thermophila. Genes \& Dev. 20: 28 33.

Liu, Y., Mochizuki, K., and Gorovsky, M.A. 2004. Histone H3 lysine 9 methylation is required for DNA elimination in developing macronuclei in Tetrahymena. Proc. Natl. Acad. Sci. 101: 1679-1684.

Liu, Y., Taverna, S.D., Muratore, T.L., Shabanowitz, J., Hunt, D.F., and Allis, C.D. 2007. RNAi-dependent H3K27 methylation is required for heterochromatin formation and DNA elimination in Tetrahymena. Genes \& Dev. 21: 1530-1545.

Madeddu, L., Gautier, M.C., Vayssie, L., Houari, A., and Sperling, L. 1995. A large multigene family codes for the polypeptides of the crystalline trichocyst matrix in Paramecium. Mol. Biol. Cell 6: 649-659.

Malone, C.D., Anderson, A.M., Motl, J.A., Rexer, C.H., and Chalker, D.L. 2005. Germ line transcripts are processed by a Dicer-like protein that is essential for developmentally programmed genome rearrangements of Tetrahymena thermophila. Mol. Cell. Biol. 25: 9151-9164.

Matzke, M. and Birchler, J. 2005. RNAi-mediated pathways in the nucleus. Nat. Rev. Genet. 6: 24-35.

Meyer, E. and Chalker, D.L. 2007. Epigenetics of ciliates. In Epigenetics (eds. C.D. Allis et al.), pp. 127-150. Cold Spring Harbor Laboratory Press, Cold Spring Harbor, NY.

Meyer, E. and Duharcourt, S. 1996. Epigenetic programming of developmental genome rearrangements in ciliates. Cell 87: 9-12.

Meyer, E. and Garnier, O. 2002. Non-Mendelian inheritance and homology-dependent effects in ciliates. Adv. Genet. 46: 305-337.

Meyer, E. and Keller, A.M. 1996. A Mendelian mutation affecting mating-type determination also affects developmental genomic rearrangements in Paramecium tetraurelia. Genetics 143: 191-202.

Mochizuki, K. and Gorovsky, M.A. 2004a. Conjugation-specific small RNAs in Tetrahymena have predicted properties of scan (scn) RNAs involved in genome rearrangement. Genes \& Dev. 18: 2068-2073.

Mochizuki, K. and Gorovsky, M.A. 2004b. Small RNAs in genome rearrangement in Tetrahymena. Curr. Opin. Genet. Dev. 14: 181-187. 
Lepère et al.

Mochizuki, K. and Gorovsky, M.A. 2005. A Dicer-like protein in Tetrahymena has distinct functions in genome rearrangement, chromosome segregation, and meiotic prophase. Genes \& Dev. 19: 77-89.

Mochizuki, K., Fine, N.A., Fujisawa, T., and Gorovsky, M.A. 2002. Analysis of a piwi-related gene implicates small RNAs in genome rearrangement in Tetrahymena. Cell 110: 689699.

Nowacki, M., Zagorski-Ostoja, W., and Meyer, E. 2005. Nowalp and Nowa2p: Novel putative RNA binding proteins involved in trans-nuclear crosstalk in Paramecium tetraurelia. Curr. Biol. 15: 1616-1628.

Nowacki, M., Vijayan, V., Zhou, Y., Schotanus, K., Doak, T.G., and Landweber, L.F. 2008. RNA-mediated epigenetic programming of a genome-rearrangement pathway. Nature 451: $153-158$.

Sambrook, J. and Russell, D.W. 2001. Molecular cloning: A laboratory manual., 3rd ed., Cold Spring Harbor Laboratory Press, Cold Spring Harbor, NY.

Skouri, F. and Cohen, J. 1997. Genetic approach to regulated exocytosis using functional complementation in Paramecium: Identification of the ND7 gene required for membrane fusion. Mol. Biol. Cell 8: 1063-1071.

Taverna, S.D., Coyne, R.S., and Allis, C.D. 2002. Methylation of histone $\mathrm{h} 3$ at lysine 9 targets programmed DNA elimination in Tetrahymena. Cell 110: 701-711.

Yao, M.C. and Chao, J.L. 2005. RNA-guided DNA deletion in Tetrahymena: An RNAi-based mechanism for programmed genome rearrangements. Annu. Rev. Genet. 39: 537-559.

Yao, M.C., Duharcourt, S., and Chalker, D.L. 2002. Genomewide rearrangements of DNA in ciliates. In Mobile DNA II (eds. N.L. Craig et al.). ASM Press, Washington, DC.

Yao, M.C., Fuller, P., and Xi, X. 2003. Programmed DNA deletion as an RNA-guided system of genome defense. Science 300: 1581-1584.

Zaratiegui, M., Irvine, D., and Martienssen, R. 2007. Noncoding RNAs and gene silencing. Cell 128: 763-776. 


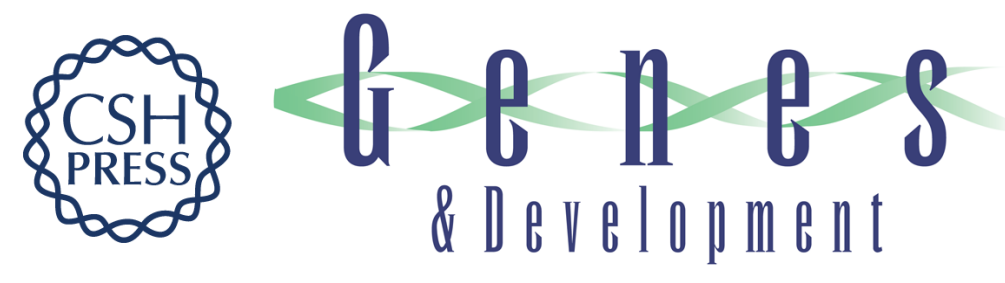

\section{Maternal noncoding transcripts antagonize the targeting of DNA elimination by scanRNAs in Paramecium tetraurelia}

Gersende Lepère, Mireille Bétermier, Eric Meyer, et al.

Genes Dev. 2008, 22:

Access the most recent version at doi:10.1101/gad.473008

Supplemental http://genesdev.cshlp.org/content/suppl/2008/05/21/22.11.1501.DC1
Material

References This article cites 42 articles, 19 of which can be accessed free at:

http://genesdev.cshlp.org/content/22/11/1501.full.html\#ref-list-1

License

Email Alerting

Receive free email alerts when new articles cite this article - sign up in the box at the top

Service

right corner of the article or click here.

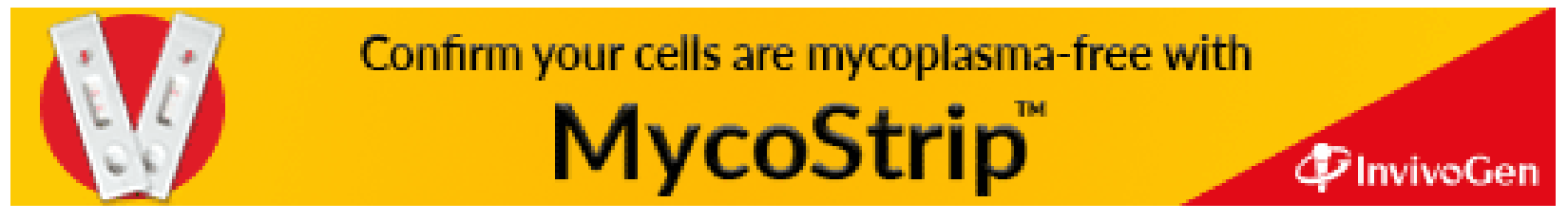

